\title{
Diagnostic challenges and management of a patient with acromegaly due to ectopic growth hormone-releasing hormone secretion from a bronchial carcinoid tumour
}

\author{
Nikolaos Kyriakakis', Jacqueline Trouillas², Mary N Dang³, Julie Lynch', \\ Paul Belchetz', Márta Korbonits ${ }^{3}$ and Robert D Murray ${ }^{1}$ \\ 1Leeds Centre for Diabetes and Endocrinology, St James's University Hospital, Leeds Teaching Hospitals NHS Trust, \\ Leeds, UK, ${ }^{2}$ Centre de Pathologie Est, Hospices Civils de Lyon, Groupement Hospitalier Est, University of Lyon, \\ Lyon, France, and ${ }^{3}$ Endocrinology, William Harvey Research Institute, Barts and The London School of Medicine \\ and Dentistry, Queen Mary University of London, London, UK
}

\author{
Correspondence \\ should be addressed \\ to R D Murray \\ Email \\ robertmurray@nhs.net
}

\section{Summary}

A male patient presented at the age of 30 with classic clinical features of acromegaly and was found to have elevated growth hormone levels, not suppressing during an oral glucose tolerance test. His acromegaly was originally considered to be of pituitary origin, based on a CT scan, which was interpreted as showing a pituitary macroadenoma. Despite two transsphenoidal surgeries, cranial radiotherapy and periods of treatment with bromocriptine and octreotide, his acromegaly remained active clinically and biochemically. A lung mass was discovered incidentally on a chest X-ray performed as part of a routine pre-assessment for spinal surgery 5 years following the initial presentation. This was confirmed to be a bronchial carcinoid tumour, which was strongly positive for growth hormone-releasing hormone (GHRH) and somatostatin receptor type 2 by immunohistochemistry. The re-examination of the pituitary specimens asserted the diagnosis of pituitary GH hyperplasia. Complete resolution of the patient's acromegaly was achieved following right lower and middle lobectomy. Seventeen years following the successful resection of the bronchial carcinoid tumour the patient remains under annual endocrine follow-up for monitoring of the hypopituitarism he developed after the original interventions to his pituitary gland, while there has been no evidence of active acromegaly or recurrence of the carcinoid tumour. Ectopic acromegaly is extremely rare, accounting for $<1 \%$ of all cases of acromegaly. Our case highlights the diagnostic challenges differentiating between ectopic acromegaly and acromegaly of pituitary origin and emphasises the importance of avoiding unnecessary pituitary surgery and radiotherapy. The role of laboratory investigations, imaging and histology as diagnostic tools is discussed.

\section{Learning points:}

- Ectopic acromegaly is rare, accounting for less than $1 \%$ of all cases of acromegaly.

- Ectopic acromegaly is almost always due to extra-pituitary GHRH secretion, mainly from neuroendocrine tumours of pancreatic or bronchial origin.

- Differentiating between acromegaly of pituitary origin and ectopic acromegaly can cause diagnostic challenges due to similarities in clinical presentation and biochemistry.

- Serum GHRH can be a useful diagnostic tool to diagnose ectopic acromegaly.

- Pituitary imaging is crucial to differentiate between a pituitary adenoma and pituitary hyperplasia, which is a common finding in ectopic acromegaly.

- Diagnosing ectopic acromegaly is pivotal to avoid unnecessary interventions to the pituitary and preserve normal pituitary function. 


\section{Background}

Acromegaly is characterised by typical skeletal changes, with coarsened facial features, pronounced growth of hands and feet, soft tissue hypertrophy and a wide range of systemic clinical features involving the cardiovascular, respiratory, gastrointestinal and metabolic systems. In the overwhelming majority of the cases, this is the result of growth hormone (GH) excess from a pituitary adenoma (1). Ectopic acromegaly is rare, accounting for less than $1 \%$ of all cases of acromegaly and is almost always the result of extra-pituitary GH-releasing hormone (GHRH) secretion (2), apart from a small number of cases describing ectopic GH production related to a neuroendocrine tumour or non-Hodgkin's lymphoma $(3,4)$. Since the isolation and characterisation of GHRH from two human pancreatic tumours in 1982 $(5,6)$, there have been a limited number of cases of ectopic acromegaly described. Adding to the existing literature, we present the clinical case of a patient who was originally thought to have a treatment-resistant GH-secreting pituitary adenoma, but subsequently was found to have ectopic GHRH secretion from a lung carcinoid tumour, resulting in pituitary hyperplasia. Highlighting the diagnostic challenges differentiating ectopic acromegaly and acromegaly of pituitary origin, we discuss the role of laboratory investigations, imaging and histology as diagnostic tools.

\section{Case presentation}

A currently 52-year-old man was diagnosed with acromegaly at age 30 , when he was noted to have typical skeletal features of acromegaly by a general surgeon who assessed the patient for abdominal pain and referred him for endocrine assessment. On further assessment, it was found that the patient had gradual weight gain and increase in the size of his hands and feet for a period of 4 years prior to diagnosis.

\section{Investigation}

The patient was diagnosed with acromegaly based on an elevated basal GH level of $181 \mathrm{IU} / \mathrm{L}$, which failed to suppress during an oral glucose tolerance test (OGTT). A pituitary CT scan was reported as showing a large tumour occupying the pituitary fossa, with some bulge into the suprasellar region and without involvement of the optic pathway. The lesion was of variable density with some overall enhancement. Results of the remaining anterior pituitary function showed a suboptimal cortisol response to the insulin tolerance test (ITT), with a basal cortisol of $459 \mathrm{nmol} / \mathrm{L}$, which failed to rise despite adequate hypoglycaemia; prolactin (PRL) 524 (normal: <600) IU/L; normal thyroid function (TSH: 0.84 (reference range: 0.2-6.0) $\mathrm{mIU} / \mathrm{L}$ and total T4: 118 (reference range: 60-140) $\mathrm{nmol} / \mathrm{L}$ ); and mildly elevated gonadotrophins (FSH: 14 IU/L, LH: 10 IU/L; no information about patient's preoperative testosterone level is available). Due to inappropriate cortisol response to the ITT, the patient was commenced on oral hydrocortisone replacement.

\section{Treatment}

Based on the OGTT results and pituitary imaging, the patient was diagnosed with acromegaly related to a pituitary adenoma and referred for trans-sphenoidal surgery. 'Complete resection of a pituitary macroadenoma' was documented in the operation note. Histological examination was reported as showing evidence of 'pituitary adenoma' comprising sheets of cells with brightly eosinophilic granular cytoplasm and moderately pleomorphic nuclei. Immunostaining demonstrated strong positivity for $\mathrm{GH}$ and prolactin and scattered positivity for LH, FSH, TSH and ACTH.

Post-operatively, the patient continued to have biochemical evidence of active acromegaly (basal GH at $109 \mathrm{mIU} / \mathrm{L}$, suppressed to $39 \mathrm{mIU} / \mathrm{L}$ during an OGTT). No significant deterioration in pituitary function was noted post-operatively (prolactin: $685 \mathrm{mIU} / \mathrm{L}, \mathrm{FSH}: 16.1 \mathrm{IU} / \mathrm{L}$, LH: 6.7 IU/L, testosterone: 8.2 (reference range: 8.0-27.0) $\mathrm{nmol} / \mathrm{L}$, TSH: $0.42 \mathrm{mIU} / \mathrm{L}$, total T4: $108 \mathrm{nmol} / \mathrm{L})$. There is no record of the patient's SHBG or free testosterone level at that time. Due to total testosterone levels close to the lower end of normal range and symptoms of erectile dysfunction, the patient was commenced on testosterone replacement therapy and remained on oral hydrocortisone post-operatively.

In view of biochemical evidence of active acromegaly, the patient was commenced on bromocriptine and was referred for conventional external beam radiotherapy, completed in June 1994 (45 Gy in 25 fractions). A subsequent GH day curve showed elevated mean GH level at $132.3 \mathrm{mIU} / \mathrm{L}$. Repeat pituitary imaging, with MRI in December 1994, showed an enlarged sella filled with homogenous soft tissue of relatively high signal on the unenhanced scan and evidence of uniform enhancement following contrast injection. However, no focal lesion was detected and no extension to the suprasellar cistern was seen. 
Bromocriptine was changed to subcutaneous octreotide $50 \mu \mathrm{g}$ three times daily due to gastrointestinal side effects. This was discontinued a few months later, due to worsening headaches and gastrointestinal symptoms. Due to ongoing clinical and biochemical evidence of active acromegaly, the patient was referred for pituitary re-exploration, two years following the original pituitary surgery. During the second trans-sphenoidal surgery, dense fibrous tissue was found in the sella and no significant resection was possible. No improvement to the GH levels was noted after the second procedure and the patient underwent a further challenge with octreotide, this time in the form of the LAR preparation, which once again was not tolerated. A follow-up pituitary MRI scan two years after the second trans-sphenoidal surgery was reported to show improved overall appearance of the sella region, with considerable reduction of the intrasellar content volume and without any focal abnormality.

Simultaneously, the patient was being pre-assessed for an orthopaedic operation due to spinal stenosis. A routine chest X-ray revealed a right lower lobe shadow. This was investigated further by a thorax CT scan, which showed an $8 \mathrm{~cm}$ tumour in the posterior segment of the right lower lobe, without evidence of lymphadenopathy or metastatic disease. A fibre-optic bronchoscopy confirmed the presence of a tumour, arising from the right posterior and right anterior basal segments, extending upwards above the level of the medial segment to the bronchus of the right lower lobe. A bronchial biopsy showed features highly suggestive of bronchial carcinoid; histological description included fragments of endobronchial mucosa within the subepithelial layer islands of tumour composed of relatively monomorphic cells with central round nuclei. Immunostaining with chromogranin was strongly positive (consistent with carcinoid tumour) and GH immunostaining was negative. GHRH staining was not available at that time and serum chromogranin was not tested. The patient underwent right lower and middle lobectomy with complete resection of the bronchial carcinoid, which resulted in rapid normalisation of GH and IGF1 levels. A pattern of the patient's GH and IGF1 levels across the different stages of his treatment and endocrine follow-up is shown in Figure 1.

The patient's case was recently re-visited and further histological examination of the bronchial carcinoid tumour and pituitary tissues conducted. Immunohistochemical study of the bronchial carcinoid samples was strongly positive for GHRH with the percentage of positive cells varying from 0 to $80 \%$ in some areas (Fig. $2 \mathrm{~A}$ ). Almost all the GHRH-positive cells presented a regular plasma

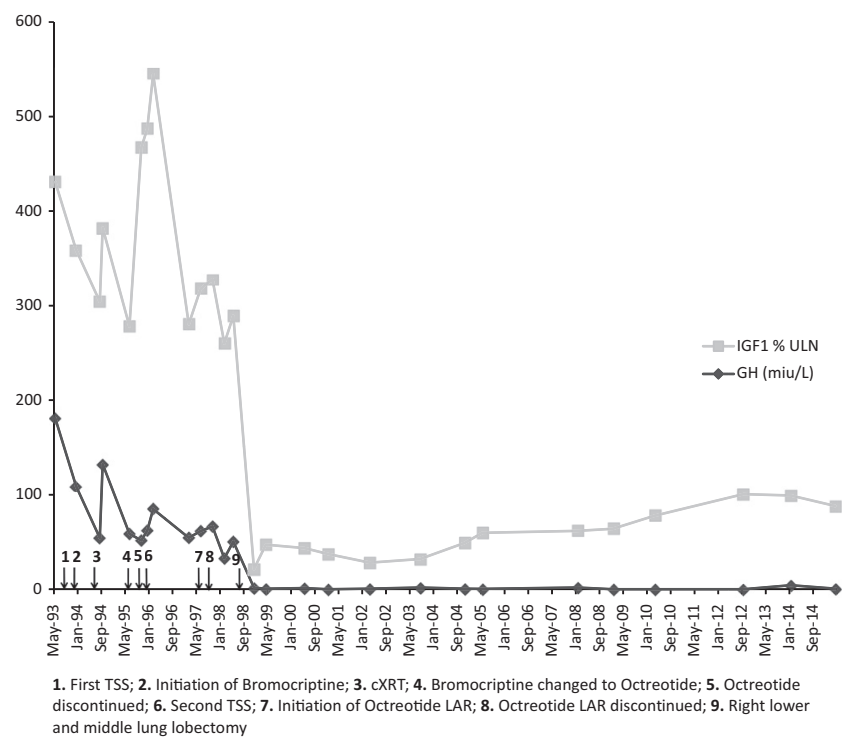

Figure 1

The graph demonstrates the trend in the patient's GH and IGF1 levels from the time of his original presentation until now. The various therapeutic interventions are also shown across the patient's timeline. A dramatic drop and normalisation of the GH and IGF1 values is seen only after the patient had lower and middle lobectomy of the right lung in 1998, which led to successful resection of a GHRH-secreting bronchial carcinoid. Both GH and IGF1 have remained within normal limits since then. IGF1 is presented as percentage of the upper limit of normal range of the IGF1 value, according to patient's age at the time of the test. GH was measured in mIU/L. TSS, trans-sphenoidal surgery; cXRT, cranial radiotherapy.

membrane reaction with antibodies against somatostatin receptors type 2 (SSTR2) (Fig. 2B). Staining was negative for GH and somatostatin receptor type 5 (SSTR5). Revision of the pituitary histology with haematoxylineosin and reticulin staining showed an arrangement of
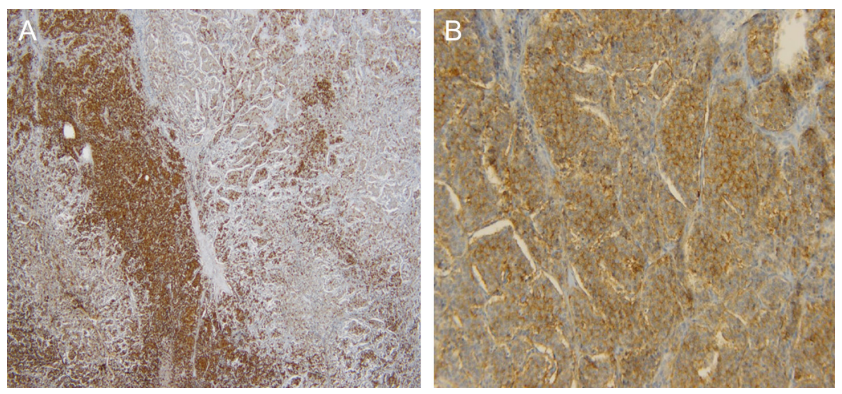

Figure 2

Immunohistochemical study of the bronchial carcinoid tumour.

(A) Typical cord-like structure in a fragment of the bronchial carcinoid. A strongly positive reaction with GHRH antibodies (polyclonal anti-GHRH from Cohen $(15,19)$, dilution $1 / 1000)$ was seen in approximately $50 \%$ of cells. The percentage varied from 0 to $80 \%$ from one area to another (10 $\times$ magnification). (B) Almost all cells presented a regular plasma membrane reaction to SSTR2 antibodies (monoclonal anti-SSTR2 from Abcam clone 109495, dilution 1/500). Some cells exhibited a cytoplasmic reaction which is considered as a background ( $100 \times$ magnification). 

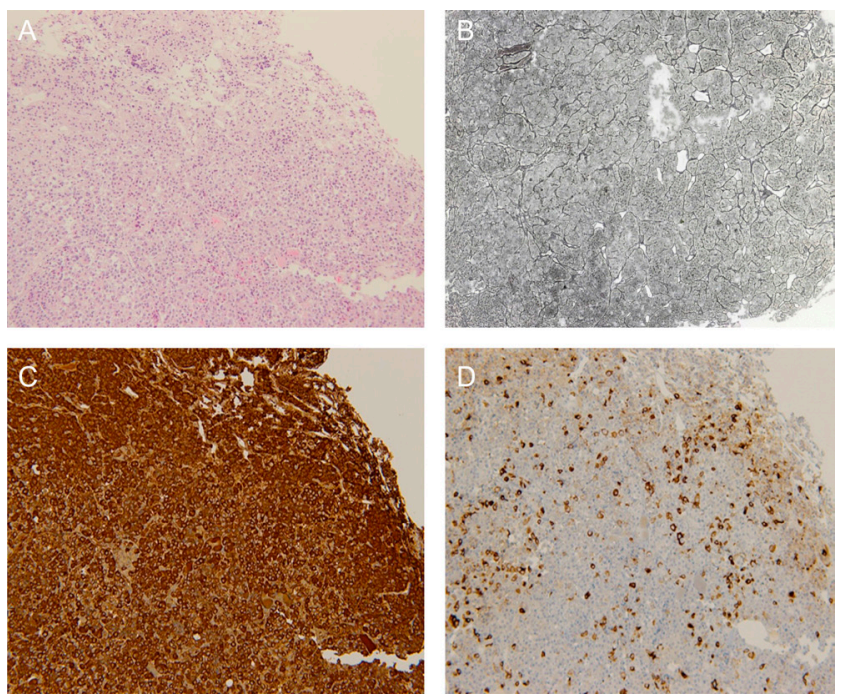

Figure 3

Immunohistochemical study of the hyperplastic pituitary gland. (A) Haematoxyline-eosine staining showing pleiomorph cells in cordonal arrangement, which is underlined by the reticulin staining (B). The cellular polymorphism of the hyperplastic pituitary is proven by the presence of $(\mathrm{C})$ numerous somatotroph cells, strongly positive with $\mathrm{GH}$ antibodies (polyclonal anti-GH from NIH, dilution 1/2000 (27), 50× magnification), and (D) scattered prolactin cells (polyclonal anti-prolactin from DAKO, dilution $1 / 2000,50 \times$ magnification). The immunostainings of figures $A, C, D$ were performed on contiguous sections. The scattered corticotroph and gonadotroph cells in this area are not shown.

cells in large cords (Fig. 3A and B), with numerous GH and prolactin strongly immunoreactive cells (Fig. 3C and D), few scattered gonadotroph cells positive for $\beta \mathrm{FSH}$ and few basophilic cells positive for ACTH. Staining for GHRH was negative in the pituitary. Based on reticulin staining and the cellular polymorphism, the pituitary histology is suggestive of pituitary hyperplasia rather than GH-PRL secreting pituitary adenoma. Following these histological findings, we have confirmed that the patient had a GHRH-secreting bronchial carcinoid with SSTR2 expression, inducing pituitary GH hyperplasia with resultant acromegaly.

\section{Outcome and follow-up}

The patient is currently 17 years post-surgical removal of the bronchial carcinoid, without evidence of acromegaly recurrence clinically or biochemically. A CT scan of thorax, abdomen and pelvis 6 years following lobectomy was negative for carcinoid recurrence. As a result of the previous pituitary interventions, the patient has developed hypopituitarism involving the ACTH, LH/FSH and TSH axes. He is on stable replacement with oral hydrocortisone, testosterone undecanoate intramuscular injections and levothyroxine and remains under routine annual follow-up.

Regarding other long-term complications of acromegaly, the patient is on lipid-lowering therapy with a statin for hypercholesterolaemia, has developed symptoms of arthropathy affecting the joints of his hands, shoulders and knees and has previously had spinal operation for spinal stenosis. An echocardiogram showed mild bi-atrial dilatation and mild mitral regurgitation, with overall preserved left ventricular function and no evidence of cardiomyopathy. Screening for hypertension, diabetes mellitus, obstructive sleep apnoea and colonic polyps has been negative.

\section{Discussion}

We have presented the case of a young male patient diagnosed late with a GHRH-secreting bronchial carcinoid tumour, 5 years after his original presentation to the endocrine services and 9 years after the onset of his symptoms of acromegaly, for which the patient underwent two pituitary surgeries and radiotherapy for presumed pituitary-related acromegaly. Differentiating between pituitary and ectopic acromegaly can impose significant diagnostic challenges. Clinically, patients present with classic symptoms and signs of acromegaly and therefore symptomatology is not helpful to distinguish between the two pathologies, unless the patient develops clinical features, which would be unexpected in an acromegalic patient and indicative of an ectopic source (i.e. respiratory wheeze, dyspnoea, flushing and symptoms related to metastatic disease).

Equally, biochemistry is similar in both pituitary and ectopic acromegaly, characterised by elevated IGF1 and GH levels, with the latter failing to suppress following an OGTT. However, serum GHRH has been proposed as a useful diagnostic tool. GHRH levels have been considered to be not only a reliable marker for the diagnosis of ectopic acromegaly (increased GHRH level are seen in ectopic acromegaly vs low levels in acromegaly of pituitary origin), but also an indicator of the disease activity following surgical treatment and a sensitive marker to detect disease recurrence (7). Nevertheless, in our case serum GHRH was not established at diagnosis. An additional diagnostic tool for ectopic GHRH secretion is the detection of GHRH-positive cells in the tumour. However, this immunocytochemistry technique is not available routinely and requires specific antibodies (which are not commercialised), good fixative of the tumour and large fragments, as in many cases the immunoreactive 
cells are grouped in islets and certain areas of the tumour may be completely negative.

Pituitary imaging does not always allow differentiation between ectopic and pituitary-related acromegaly. Normal volume pituitary or global pituitary hyperplasia are the expected findings from pituitary imaging in ectopic acromegaly. However, in a review of 63 pituitary MRI scans of patients with ectopic acromegaly, 12 were reported as showing normal appearances of the pituitary gland, 38 as pituitary hyperplasia, while 13 were reported as pituitary adenomas, suggesting that the differentiation between pituitary hyperplasia and adenoma based on imaging is not always possible (7). This highlights the importance of an experienced neuroradiologist for the interpretation of pituitary imaging, as this can be the only indication of the alternative diagnosis, which can significantly alter and determine patient management. In our case, increased pituitary volume with suprasellar extension was found on the original pituitary imaging, which in combination with the clinical picture and the biochemical results were thought to be indicative of a typical case of acromegaly related to pituitary macroadenoma.

The histological differential diagnosis between GH hyperplasia and a true GH-PRL tumour is also very difficult. It is based on the reticulin stain showing a cordonal arrangement of the cells, which is exceptional in GH tumours. The cellular polymorphism with FSH and ACTH strongly positive cells scattered in the whole fragments is also in favour of hyperplasia. Indeed, plurihormonal GH-PRL-FSH adenomas are exceptionally rare in sporadic somatotrophinomas. It has been described in multiple endocrine neoplasia 1 (MEN1)-related pituitary adenomas where pituitary hyperplasia could also be found (8).

Extra-pituitary GHRH tumours have been reported to be of a considerable size, ranging between 1 and $25 \mathrm{~cm}$ and therefore can often be detected by conventional body imaging (CT or MRI scan of thorax, abdomen and pelvis) $(2,7)$. Somatostatin receptor scintigraphy is an alternative option for the localisation of these tumours as they are usually well-differentiated and express somatostatin receptors, such as in our case. This is particularly useful for tumours of small size, when initial imaging has not been successful to localise the tumour, and also when evaluation for metastatic disease is needed (7). The tumour characteristics in our case were compatible with those reported in the literature. Our patient had a sizeable bronchial carcinoid tumour measuring $8 \mathrm{~cm}$, which was positive for SSTR2.

It has been suggested that an association may exist between ectopic acromegaly and MEN1. This particularly applies to GHRH-secreting pancreatic tumours, as MEN1 syndrome was found in 19 out of 25 cases reported (7). Our patient did not have a pancreatic tumour and had normal calcium levels, but was tested for the MEN1 gene, which was found to be negative.

Treatment of ectopic acromegaly is mainly surgical and involves resection of the responsible tumour. When this is contraindicated or in case of metastatic disease, somatostatin analogues can be an alternative option to achieve biochemical disease remission with normalisation of GH and IGF1 levels. Reduction, but not complete normalisation of serum GHRH is usually seen with somatostatin analogue treatment, suggesting that these agents have a dual effect; however, biochemical control of acromegaly is mainly via the effect of these agents on the pituitary gland, reducing GH release (7).

Prognosis is overall favourable for patients with ectopic acromegaly following surgical removal of the responsible tumour. A cure rate of $87 \%$ after a median follow-up of 2 years was reported by Losa et al. in a series of 23 cases (9), while a survival rate of $85 \%$ after a 5 -year median follow-up was reported in a French series of 21 cases (10). Despite the original delay in the diagnosis, our patient had a successful outcome following resection of the bronchial carcinoid without any evidence of disease activity or recurrence after a 17-year follow-up period.

Diagnosing ectopic acromegaly is pivotal in the management of the patient, as excision of the responsible tumour is usually curative, whilst avoiding unnecessary interventions to the pituitary preserves normal pituitary function. Ectopic acromegaly should be considered in patients with clinical manifestations of acromegaly when pituitary imaging fails to demonstrate a discrete adenoma; when pituitary histology is not compatible with an adenoma (i.e. preservation of the reticulin network or immunostaining positive for a variety of pituitary hormones); or in patients with resistant acromegaly despite multimodality treatment. This case adds to the existing literature of clinical cases of ectopic acromegaly, providing histological pictures of the pituitary hyperplasia in particular and highlights the diagnostic challenges encountered in clinical practice in order to differentiate between ectopic acromegaly and acromegaly of pituitary origin.

Declaration of interest

The authors declare that there is no conflict of interest that could be perceived as prejudicing the impartiality of the research reported. 


\section{Funding statement}

This research did not receive any specific grant from any funding agency in the public, commercial or not-for-profit sector

\section{Patient consent}

Written informed consent for publication of the submitted article and accompanying images has been obtained from the patient.

\section{Author contribution statement}

N Kyriakakis, M Dang, M Korbonits, J Lynch and R D Murray were responsible for data collection and analysis. J Trouillas interpreted the histological data. N Kyriakakis drafted the manuscript. N Kyriakakis, M Korbonits, J Trouillas, P Belchetz and R D Murray reviewed and edited the manuscript.

\section{References}

1 Melmed S 2006 Medical progress: acromegaly. New England Journal of Medicine 355 2558-2573. (doi:10.1056/NEJMra062453)

2 Ghazi AA, Amirbaigloo A, Dezfooli AA, Saadat N, Ghazi S, Pourafkari M, Tirgari F, Dhall D, Bannykh S, Melmed S, et al. 2013 Ectopic acromegaly due to growth hormone releasing hormone. Endocrine $\mathbf{4 3}$ 293-302. (doi:10.1007/s12020-012-9790-0)

3 Melmed S, Ezrin C, Kovacs K, Goodman RS \& Frohman LA 1985 Acromegaly due to secretion of growth hormone by an ectopic pancreatic islet-cell tumor. New England Journal of Medicine 312 9-17. (doi:10.1056/NEJM198501033120103)
4 Beuschlein F, Strasburger CJ, Siegerstetter V, Moradpour D, Lichter P, Bidlingmaier M, Blum HE \& Reincke M 2000 Acromegaly caused by secretion of growth hormone by a non-Hodgkin's lymphoma New England Journal of Medicine 342 1871-1876. (doi:10.1056/ NEJM200006223422504)

5 Guillemin R, Brazeau P, Böhlen P, Esch F, Ling N \& Wehrenberg WB 1982 Growth hormone-releasing factor from a human pancreatic tumor that caused acromegaly. Science 218 585-587. (doi:10.1126/ science. 6812220

6 Rivier J, Spiess J, Thorner M \& Vale W 1982 Characterization of a growth hormone-releasing factor from a human pancreatic islet tumor. Nature 300 276-278. (doi:10.1038/300276a0)

7 Borson-Chazot F, Garby L, Raverot G, Claustrat F, Raverot V, Sassolas G \& the GTE Group 2012 Acromegaly induced by ectopic secretion of GHRH: a review 30 years after GHRH discovery. Annales d'Endocrinologie 73 497-502. (doi: 10.1016/j. ando.2012.09.004)

8 Trouillas J, Labat-Moleur F, Sturm N, Kujas M, Heymann MF, Figarella-Branger D, Patey M, Mazucca M, Decullier E, Vergès B, et al. 2008 Pituitary tumors and hyperplasia in multiple endocrine neoplasia type 1 syndrome (MEN1): a case-control study in a series of 77 patients versus 2509 non-MEN1 patients. American Journal of Surgical Pathology 32 534-543. (doi:10.1097/ PAS.0b013e31815ade45)

9 Losa M, Schopohl J \& von Werder K 1993 Ectopic secretion of growth hormone-releasing hormone in man. Journal of Endocrinological Investigation 16 69-81. (doi:10.1007/BF03345835)

10 Garby L, Caron P, Claustrat F, Chanson P, Tabarin A, Rohmer V, Arnault G, Bonnet F, Chabre O, Christin-Maitre S, et al. 2012 Clinical characteristics and outcome of acromegaly induced by ectopic secretion of growth hormone-releasing hormone (GHRH). A French nationwide series of 21 cases. Journal of Clinical Endocrinology and Metabolism 97 2093-2104. (doi:10.1210/jc.2011-2930)

Received in final form 16 October 2016

Accepted 13 December 2016 